$\begin{gathered}\text { ADVANCE RESEARCH JOURNAL OF SOCIAL SCIENCE } \\ \text { Aolume 10 | Issue 2 | December, 2019 | 75-78 } \\ \text { A ISSN-0976-5611 }\end{gathered}$
DOI: 10.15740/HAS/ARJSS/10.2/75-78

\title{
Transition of kani community in Thiruvananthapuram district
}

Sanitha Beevis

Department of History, Iqbal College, Peringammala, Trivandrum (Kerala) India

(Email : sanithadanish@gmail.com)

\section{ARTICLE INFO :}

Received

Accepted

19.08 .2019

28.11.2019
KEY WORDS :

Kani community

HOW TO CITE THIS ARTICLE :

Beevis, Sanitha (2019). Transition of kani community in Thiruvananthapuram district. Adv. Res. J. Soc. Sci., 10 (2) : 75-78, DOI: 10.15740/HAS/ARJSS/10.2/75-78. Copyright@ 2019 : Hind Agri -Horticultural Society 